\title{
TITLE:
}

\section{RIG-I-like receptors: cytoplasmic sensors for non-self RNA.}

$\operatorname{AUTHOR}(S)$ :

Kato, Hiroki; Takahasi, Kiyohiro; Fujita, Takashi

\section{CITATION:}

Kato, Hiroki ... [et al]. RIG-I-like receptors: cytoplasmic sensors for nonself RNA.. Immunological reviews 2011, 243(1): 91-98

\section{ISSUE DATE:}

2011-08-26

URL:

http://hdl.handle.net/2433/196849

\section{RIGHT:}

This is the peer reviewed version of the following article: Kato, H. Takahasi, K. and Fujita, T. (2011), RIG--like receptors: cytoplasmic sensors for non-self RNA. Immunological Reviews, 243: 91-98, which has been published in final form at http://dx.doi.org/10.1007/978-1-62703-342-8_5; この論文は出版社版でありません。引用の際には出版社版をご確認じ 利用ください。; This is not the published version. Please cite only the published version. 
Hiroki Kato

Kiyohiro Takahasi

Takashi Fujita

\section{RIG-I-like receptors: cytoplasmic sensors for non-self RNA}

Authors' addresses

Hiroki Kato ${ }^{1,2}$, Kiyohiro Takahasi ${ }^{1,3}$, Takashi Fujita ${ }^{1,2}$

${ }^{1}$ Laboratory of Molecular Genetics, Institute for Virus,

Research, Kyoto University, Kyoto, Japan.

${ }^{2}$ Laboratory of Molecular Cell Biology, Graduate School of;

Biostudies, Kyoto University, Kyoto, Japan.

${ }^{3}$ Institute for Innovative NanoBio Drug Discovery and

Development, Graduate School of Pharmaceutical Sciences,

Kyoto University, Kyoto, Japan.

Correspondence to:

Takashi Fujita

Laboratory of Molecular Genetics

Institute for Virus Research, Kyoto University

Shogoinkawahara-cho, Sakyo-ku

Kyoto 606-8507

Japan

Tel.: +81 57514031

Fax: +81757514031

e-mail: tfujita@virus.kyoto-u.ac.jp

Conflict of interest

The authors have no conflicts of interest to declare.

Immunological Reviews 2011

Vol. 243: 1-8

Printed in Singapore. All rights reserved

CC 2011 John Wiley \& Sons A/S

Immunological Reviews

0105-2896
Summary: Viral infection results in the generation of non-self RNA species in the cells, which is recognized by retinoic acid inducible gene-I-like receptors (RLRs), and initiates innate antiviral responses, including the production of proinflammatory cytokines and type I interferon. In this review, we summarize reports on virus-specificity of RLRs, structures of non-self RNA patterns, structural biology of RLRs, and the signaling adapter molecules involved in antiviral innate immunity.

Keywords: antiviral innate immunity, type I interferon, RIG-I-like receptors, non-self RNA

\section{RIG-I-like receptors (RLRs)}

Pattern recognition receptors (PRRs) are essential in innate immunity. Major PRRs are Toll-like receptors (TLRs), retinoic acid inducible gene-I (RIG-I)-like receptors (RLRs), and nucleotide oligomerization domain (Nod)-like receptors (NLRs) (1-3). TLR3, TLR7, TLR8, and TLR9 detect viral 2 nucleic acids in endosome (4-8). These receptors essentially sense extracellular molecules incorporated into endosome by endocytosis. These sensors detect viral nucleic acids, released from virus-infected cells, and activate subsequent immune reactions. RLRs detect replicating viruses in cytoplasm, particularly at early phase of viral infection. Some of the NLRs sense viral infection to initiate inflammatory responses. Activation of innate immune responses lead to the induction of type I and III interferon (IFN) and inflammatory cytokines, whose antiviral activity blocks viral replication and facilitate the activation of antigen-presenting cells to activate antigen-specific immunity to eradicate the viral pathogens.

RLRs include RIG-I, MDA5, and LGP2, all of which contain a DExD/H box helicase domain $(9,10)$. The helicase domain retains catalytic activity to unwind double stranded RNA (dsRNA) in an adenosine triphosphate (ATP) hydrolysis- 
dependent manner (11); however, unwinding per se may not be inducing antiviral activity. RIG-I and MDA5 contain tandem caspase recruitment and activation domain (CARD) at their amino-termini. Forced over expression of the CARD alone is sufficient to drive antiviral signaling resulting in IFN production, showing that this domain is responsible for signaling. Indeed, the CARD of RIG-I interacts with another CARD-containing molecule, interferon- $\beta$ promoter stimulator 1(IPS-1) (also termed as MAVS, VISA, and Cardif) (12-15). Over expression of full-length RIG-I exhibits low basal activity. Furthermore, although IFN treatment strongly induces RIG-I protein, IFN treatment alone does not activate IFN genes. Thus, an autorepression model has been proposed. It was demonstrated that a region of RIG-I acts as repression domain, which functions in cis as well as trans. LGP2 also retains repression domain; however, the corresponding region of MDA5 does not exhibit repression function. The C-terminus of RLRs consists of a domain [C-terminal domain (CTD) ] and is responsible for binding with dsRNA. It has been hypothesized that binding of viral RNA with RIG-I induces conformational change in the presence of ATP to unmask CARD, but no report has experimentally demonstrated the conformational change so far.

\section{Recognition of RNA viruses by RLRs}

Based on the gene-targeting studies, it has been revealed that RLRs are essential for the production of type I IFNs and proinflammatory cytokines, such as interleukin-6 (IL-6), in response to viral infection. Double knockout cells for RIG-I and MDA5 produce no significant IFN upon viral infection, showing that these receptors are essential for sensing cytoplasmic viral RNA (16).

Cells lacking RIG-I are defective in producing type I IFNs and inflammatory cytokines in response to various RNA viruses including Newcastle disease virus (NDV) (Paramyxoviridae), Sendai virus (SeV) (Paramyxoviridae), vesicular stomatitis virus (VSV) (Rhabdoviridae), influenza A virus (Orthomyxoviridae), or Japanese encephalitis virus (JEV) (Flaviviridae), while MDA5-deficient cells respond normally to these viruses $(16,17)$. In contrast, the production of IFN in response to several picornaviruses, including encephalomyocarditis virus (EMCV), Mengo virus, and Theiler's virus, is abrogated in MDA5-deficient cells but not in cells lacking RIG-I $(17,18)$. Murine norovirus-1 (Caliciviridae) and murine hepatitis virus (Coronaviridae) are recognized by MDA5 $(19,20)$. In addition to JEV, another flavivirus, hepatitis C virus (HCV) is also recognized by RIG-I, while both RIG-I and
MDA5 likely recognize Dengue virus and West Nile virus (Flavivirus), redundantly (21-23). A vaccine strain of measles virus (Paramyxoviridae) activates IFN through the activation of both RIG-I and MDA5, whereas the wildtype measles virus fails to induce type I IFN production (24). Reovirus, whose genome consists of segmented dsRNA, induces IFN production mainly through MDA5 with minor contribution from RIG-I (25).

Over expression of LGP2, which lacks CARD, resulted in diminished IFN production by virus (10). However, LGP2 knockout cells exhibited attenuated IFN production upon infection of VSV or EMCV (26), suggesting that LGP2 cooperates with either RIG-I or MDA5 to sense viral RNA under physiological conditions. Influenza A virus-induced IFN production was normal in LGP2-deficient cells, suggesting that LGP2 specifically senses particular RNA patterns.

A DNA virus, Epstein-Barr virus (EBV), is reported to produce small RNAs that induce RIG-I-mediated IFN responses (27). Another DNA virus, Herpes simplex virus (HSV), is also reported to activate both RIG-I- and MDA5-dependent IFN responses via dsRNA produced during its replication (28).

\section{RNA ligands of RLRs}

\section{Polyriboinosinic:polyribocytidylic acid}

Polyriboinosinic:polyribocytidylic acid (polyI:C), a synthetic dsRNA, which was selected as a potent non-viral IFN inducer, acts as a ligand for TLR3 and RLRs $(4,25)$. Although the mechanism underlying is not known, poly I:C exhibits highest activity among the possible combinations of RNA homopolymer duplexes. Poly I:C is generated by annealing poly I and poly $\mathrm{C}$, which are synthesized by polynucleotide phosphorylase. This enzyme catalyzes the polymerization of nucleotide diphosphate, thus poly I:C harbors a $5^{\prime}$-diphosphate (29).

Two groups demonstrated that MDA5 is essential for poly I:C-mediated IFN production by gene targeting $(16,18)$. The specificity originates from the length of dsRNA rather than base composition (25). Commercial poly I:C runs as dsRNA of 4-8 kbp in gel electrophoresis. Partial digestion of the poly I:C with a dsRNA specific endonuclease, RNaseIII, led to the generation of trimmed poly I:C of about $300 \mathrm{bp}$. This short poly I:C activates RIG-I. Interestingly, short poly I:C fails to activate MDA5, and long poly I:C is incapable of activating RIG-I, although both of these bind to long and short poly I:C. Therefore, both RIG-I and MDA5 are necessary to sense various length of dsRNA. The precise mechanism of size discrimination by RIG-I and MDA5 is not known. 


\section{5' tri-phosphate RNAs}

RNA transcribed from DNA template by T7 RNA polymerase is a strong inducer of type I IFN (30). Further studies revealed that $5^{\prime}$-triphosphate containing RNA, such as genomes of most RNA viruses, and in vitro transcripts are selectively recognized by RIG-I $(31,32)$. Since the $5^{\prime}$-triphosphate moieties of most host transcripts are removed by adding 7-methyl-guanosine cap (in the case of mRNA) or by processing (tRNA and rRNA), these self-RNAs are refractory to detection by RIG-I. However more recently, it was revealed that $5^{\prime}$-triphosphate per se is not sufficient for RIG-I activation. This is consistent with observations that dsRNA without $5^{\prime}$-triphosphate can be recognized by RIG-I particularly for long dsRNA. Therefore, dsRNA structure is prerequisite for RIG-I activation and $5^{\prime}$-triphosphate facilitates the recognition of short dsRNA. In addition, removal of $5^{\prime}$-triphosphate is a viral strategy to avoid recognition by RIG-I. A viral peptide, VPg, is covalently attached to the $5^{\prime}$ end of Picornaviridae RNA, thus lacking 5'-triphosphate (33). Hantaan virus (HTNV), Crimean-Congo hemorrhagic fever virus (CCHFV) (Bunyaviridae), and Borna disease virus (BDV) (Bornaviridae) do not trigger RIG-I-mediated IFN responses, because the $5^{\prime}$-triphosphate structure of these viral genomic RNA is removed by processing (34).

Transfection of AT-rich dsDNA (typically a synthetic polydAdT:polydAdT) results in the type I IFN induction(35). It was revealed that polydAdT:polydAdT is transcribed by host DNA-dependent RNA polymerase III and that the resultant $5^{\prime}$-triphosphate dsRNA is detected by RIG-I $(36,37)$. The polymerase III-mediated mechanism may explain IFN induction by some DNA viruses or intracellular bacteria.

Nallagatla et al. suggested that the $5^{\prime}$ tri-phosphate signature is sensed by protein kinase $\mathrm{R}$ (PKR) in addition to dsRNA (38). Further studies are required to elucidate the mechanism by which the $5^{\prime}$ tri-phosphate signature activates both RIG-I and PKR and to understand its biological significance.

\section{Viral RNA ligand for RLR}

Garcin's group (39) nicely showed this dsRNA-dependent activation of RIG-I, using a SeV infection. They generated SeV expressing GFP mRNA or antisense GFP mRNA and performed co-infection of two types of SeV. They found that the resulting capped dsRNA of GFP sequences induced an IFN response that was dependent on RIG-I. In addition to engineered SeV, natural VSV, a ssRNA virus recognized by RIG-I, was shown to produce dsRNA in infected cells (25). Induction of IFNs by
RNAs from VSV-infected cells was impaired by disrupting dsRNA, suggesting the presence of dsRNA in VSV-infected cells is important for RIG-I mediated IFN responses. The fragments produced by VSV were about 2-2.5 kbp much shorter than the length of the $11 \mathrm{~kb}$ VSV genomic RNA, suggesting that these dsRNA were not derived from replication intermediates of VSV. Rather, the dsRNA maybe be derived from defective interfering (DI) particles, whose snap-back dsRNAs are reported to be about $2.2 \mathrm{kbp}$, generated in VSV-infected cells, although the viral dsRNA signiture recognized by RIG-I remains to be further characterized. Recently, panhandle structure of the influenza virus genome is also reported as RIG-I ligand (40). It is of interest to clarify which ligand is the major source of RIG-I-dependent IFN production in individual viral infection.

IFN production induced by long segments purified from the reovirus genome (about $3.9 \mathrm{kbp}$ ) is impaired in MDA5deficient cells (25). Further, the very large RNA species (stuck at the top of the gel during electrophoresis) generated by EMCV or vaccinia virus infection is a specific ligand for MDA5 (41). Although the precise structure of the RNA (termed RNA web), is not known, a highly ordered structure is critical for this activity, as heat treatment inactivated the complex.

\section{RNaseL-cleaved RNA}

RNaseL is an endonuclease thought to cleave viral RNA. Silverman's group (42) showed that RNaseL-deficient cells showed attenuated induction of IFN in response to poly I:C or Sendai virus. In addition, in the infection with EMCV and Sendai virus, RNaseL-deficient mice showed impaired IFN production in sera. The RNaseL cleavage products activate RIG-I and MDA5, leading to production of type I IFNs. This mechanism potentially generates RLR ligands from self-RNA as well as viral RNA.

\section{Poly-U/UC rich RNA}

Infection with HCV is known to be regulated by hepatic immune defense triggered by RIG-I. Mapping of the HCV genome for RIG-I activation revealed that $5^{\prime}$-triphosphate signature was necessary but not sufficient for the activation of RIG-I and that a polyuridine motif (poly-U/UC-rich region) at the $3^{\prime}$-non-translated region is critical for efficient activation of RIG-I (43). They extended their finding and found that 5'-triphosphate genomic poly-U/A-rich RNA motifs within the rabies virus leader sequence, Ebola virus $3^{\prime}$ region, and measles virus leader sequence were also important for RIG-I 
activation (43). However, GC-rich RNA motifs failed to induce the signaling (43). Thus, A/U composition and poly$\mathrm{U}$ motifs are possible determinants of viral RNA recognition by antiviral innate immunity.

\section{Structure of RLRs}

Two groups independently identified the RNA recognition domain that is located to the C-terminus to the helicase domain CTD. Takahasi et al. (11) showed that RIG-I CTD specifically binds dsRNA and 5'-triphosphate RNA and solved its solution structure. Furthermore, these studies revealed that large positive charged surface that locates the center of the structure is the RNA binding site by NMR titration (11). Cui et al. also identified the domain and solved the crystal structure (44). The structures of MDA5 and LGP2 CTDs were also solved and their structural features are very similar to RIG-I CTD (45). Recent studies have solved the crystal structures of RIG-I CTD bound to both blunt ended double-strand RNA and dsRNA with 5'-triphosphate (46-48). Both CTD structures showed that the same basic surface revealed in previous studies was essential for the RNA recognition. However, two different ended RNAs bound RIG-I CTD in different orientations (46-48). The complex structure of LGP2 bound to the blunt end of dsRNA was also solved by x-ray crystallography, but the orientation of the RNA in the complex was also different from the RNAs bound to the RIG-I CTD (49). The biological significance of these co-crystals requires further investigation because the biological activity of the RNA used for crystal is not well investigated, particularly the presence or absence of 5 -triphosphate. These observations imply that RIG-I specifically recognizes the end structure of viral RNA, especially blunt end dsRNA or 5' -triphosphate structure. However, it has been controversial if end structure affects recognition by RIGI $(11,45,46)$. In addition, AFM analysis suggests that RIG-I aggregates around the short poly $\mathrm{I}$ : $\mathrm{C}$, excluding the possibility of end-specific binding (25). For certain, better understanding of the activation of the RIG-I/RNA complex awaits further structural elucidation of inactive (free RIG-I in closed structure) and activated (RIG-I/RNA complex in the presence of ATP) complexes.

\section{RLR signaling and its regulation}

Upon binding to viral RNA in the presence of ATP, RIG-I changes its conformation to expose the CARD. The activated RIG-I forms oligomer, which allows the CARDs of RIG-I to interact with a CARD-containing adapter, IPS-1, which is localized on the outer membrane of the mitochondrion. MDA5 also transduces signaling via IPS-1. IPS-1 subsequently activates two IKB kinase (IKK)-related kinases, IKK-I, and TANK-binding kinase 1 (TBK1) via tumor necrosis factor (TNF) receptor-associated factor 3 (TRAF3) (50-52). These kinases phosphorylate IRF3 and IRF7, resulting in their trans-

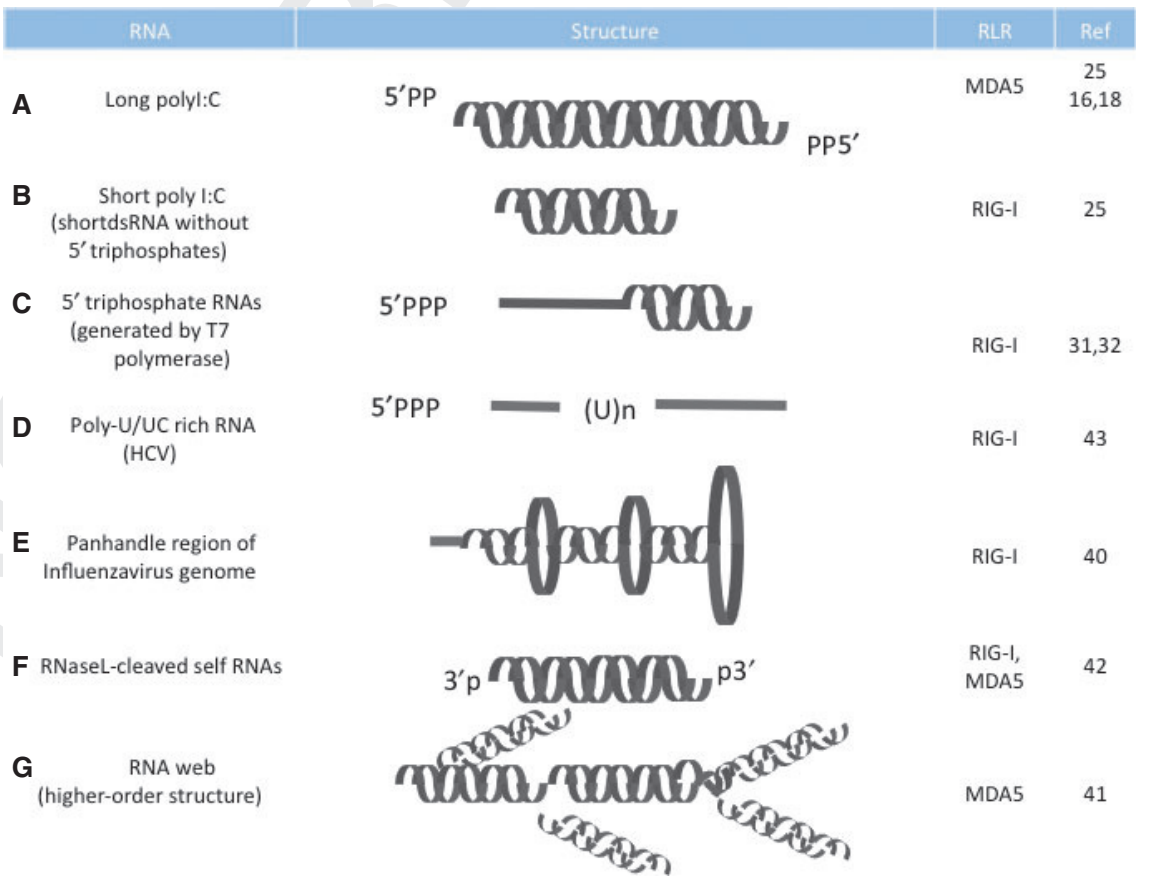

Fig. 1. RNA patterns and their recognition by RLRs. Summary of synthetic and viral RNA patterns and the RLRs recognizing them. 
location from the cytoplasm into the nucleus, which activates transcription of genes encoding type I IFNs and IFN-inducible genes $(53,54)$. In addition, IPS- 1 activates NF- $\mathrm{KB}$ via a FADD and caspase- $8 / 10$ pathway to regulate the expression of proinflammatory cytokine genes (55).

Many molecules have been reported to modulate the RLRmediated signaling pathway (described above) through direct interaction with RLRs or with signaling molecules. For instance, Src homology 2 (SH2) domain-containing phosphatase-1 (SHP-1) is reported to activate RIG-I-mediated signaling by binding directly to RIG-I (56). Dihydroacetone kinase (DAK) was identified as a MDA5-interacting protein and was shown to negatively regulate MDA5 (57). NLRX1 (also known as NOD9) is localized to the mitochondrial outer membranes and is suggested to function as a negative regulator of IPS-1 via a direct interaction (58). Suppressor of IKKe (SIKE) forms a complex with TBK1/IKKe and inhibits the interaction of RIG-I or IRFs with TBK1/IKKe in the basal condition, while viral infection leads to dissociation of SIKE from TBK/IKKe (59). Molecules involved in the autophagic machinery, Atg5-atg12, are reported to inhibit the interaction between RIG-I and IPS- 1 by association with the CARD of RIG-I and with IPS-1 (60). Recent studies from two independent groups $(61,62)$ identified the same signal-modulator molecule, designated as stimulator of IFN genes (STING), also called mediator of IRF-3 activation (MITA). STING is reported to localize to the endoplasmic reticulum (ER) membrane, and STING interacts with RIG-I (but not MDA5), and SSR2/TRP $\beta$ complex, required for protein translocation across the ER membrane. The other group reported that STING is expressed on the outer membrane of mitochondrion, where it interacts directly with IPS- 1 and IRF-3 and positively regulates the virus-dependent recruitment of TBK1 to the IPS-1 complex on mitochondrion.

Ubiquitination of RLRs is reported to control their signals both positively and negatively. The CARDs of RIG-I undergo Lys63-linked ubiquitination by tripartite motif 25 (TRIM 25), and this ubiquitination is necessary for efficient activation of RIG-I mediated signaling (63). Interestingly, MDA5 does not undergo ubiquitination by TRIM25, suggesting that CARDs of RIG-I and MDA5 are differentially regulated. A tumor suppressor, CYLD (cylindomatosis), is reported to negatively regulate

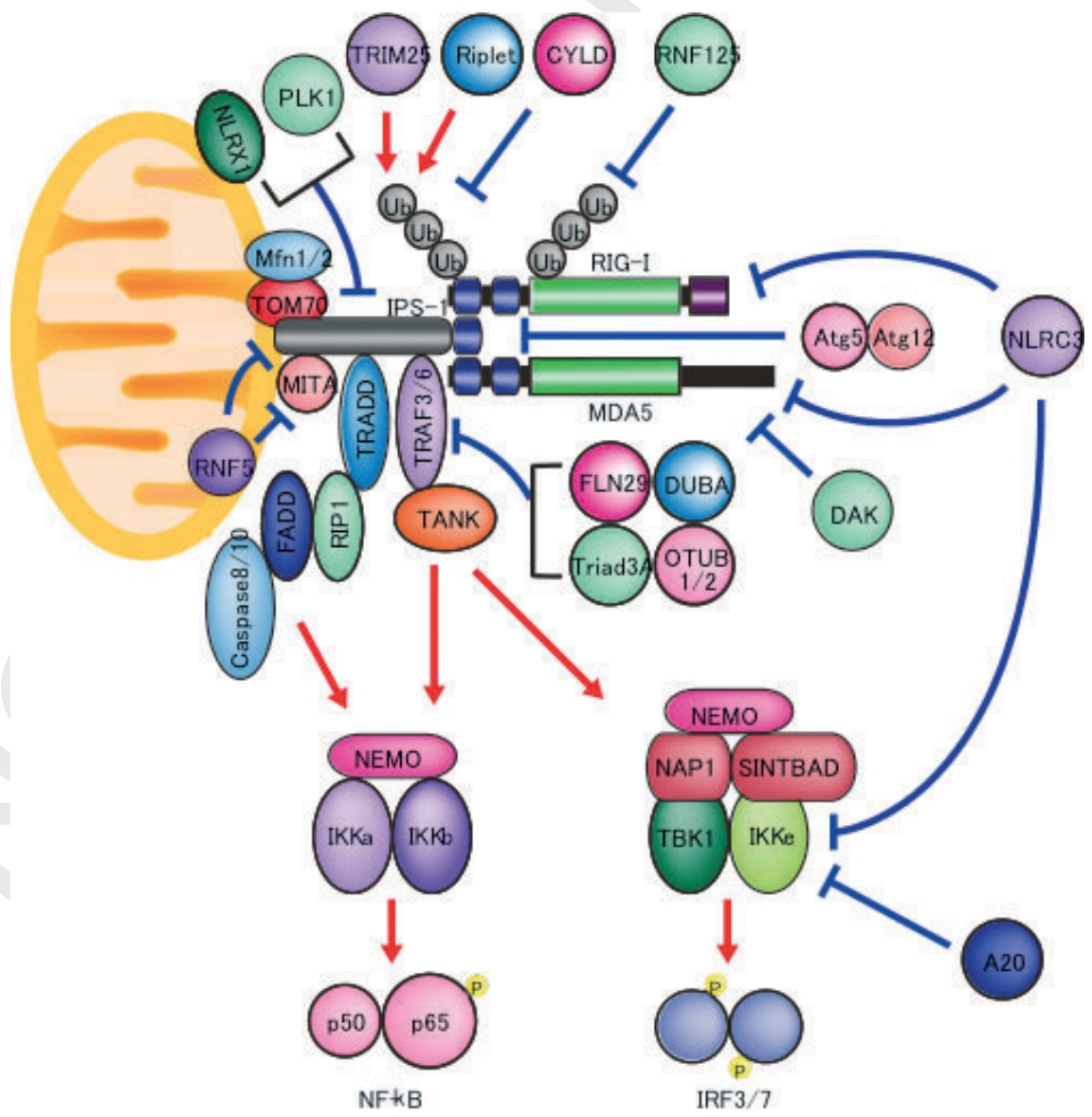

Fig. 2. RLR-mediated signaling and adapter molecules. Positive and negative regulation is indicated by red and blue lines, respectively. 
the RIG-I-mediated signaling by deubiquitinating the TRIM25-mediated ubiquitination of RIG-I (64). Another E3 ubiquitin ligase RNF135/Riplet is shown to promote Lys63linked ubiquitination of the C-terminal region of RIG-I, which results in activation of IFN promoter (65). RIG-I also undergoes Lys48-linked polyubiquitination by the E3 ubiquitin ligase RNF125, leading to its proteasomal degradation, which inhibits aberrant activation of RIG-I mediated signaling. Further, RNF125 conjugates ubiquitin to MDA5 as well as IPS1 , which results in suppressing the functions of these proteins (66). It was also recently shown that REUL is an E3 ubiquitin ligase of RIG-I and specifically stimulates RIG-I-mediated IFN signaling (67). The Lys 154, 164, and 172 residues of the RIG-I CARD domain are critical for efficient REUL-mediated ubiquitination, as well as the ability of RIG-I to induce activation of the IFN- $\beta$ promoter. ISGylation also modulates RLRsignaling. RIG-I is ISGylated by the ubiquitin-like protein, ISG15, which regulates the activity of RNF125 together with UbcH8, an E2 ubiquitin-conjugating enzyme (68). UbcH8 suppresses ubiquitination of RIG-I by RNF125, and the suppression by UbcH8 is relieved by ISG15.

Artificial aggregation of IPS-1 in the cytoplasm is sufficient for activation of antiviral signaling (69), suggesting that the activity of IPS- 1 is regulated by its oligomerization induced by physical interaction of CARD of RIG-I or MDA5. Interestingly, IPS-1 aggregation is observed in cells stably expressing Flagtagged IPS-1 upon infection by various viruses or 5'-triphosphate RNA. Normally IPS-1 is localized on the outer membrane of the mitochondrion, which may restrict its movement. The IPS-1 aggregation requires mitofusin 1 , a mitochondrial regulator for its fusion (70). Mitofusin 1 is also reported to be critical for mitochondrial elongation induced by certain strains of Sendai virus. These results suggest that the activity of IPS-1 requires dynamic fission and fusion of mitochondrion to facilitate efficient signaling by its oligomerization. However, there are contradictory reports on the function of mitochondrion and mitofusins (71).

In addition to host regulatory factors mentioned above, viruses acquire means to evade antiviral immunity of the host. Modification of viral RNA and assembly of nucleocapsid are major strategies to escape from detection by RLRs. Viral nonstructural (NS) proteins often participate in the counteraction of antiviral cascades. A large number of viral inhibitory proteins have been identified that block RLR signaling at various levels (reviewed in 72, 73).

\section{Conclusions and perspectives}

Cumulative studies based on RLR knockout mice/cells and various viruses revealed the mechanism by which RLRs sense viral invasion as well as viral strategies to evade antiviral responses mediated by RLRs. As described in this review, it is clear that RIG-I and MDA5 are essential sensors for RNA viruses and that these helicases discriminate self and non-self RNAs by precise recognition of virus-specific RNA signatures. However, elucidation of many signaling regulators has made the picture more complicated than previously appreciated. Certainly, many interesting topics remain to be clarified, including identification of the precise RNA ligands of RLRs during viral infection, the reason why IPS-1 localizes to the mitochondrion, and elucidation of the molecular structure of inactive and active RIG-I.

\section{References}

1. Takeuchi O, Akira S. Pattern recognition receptors and inflammation. Cell 2010;140:805-820

2. Fujita T. A nonself RNA pattern: tri-p to panhandle. Immunity 2009;31:4-5

3. Beutler B, et al. Genetic analysis of resistance to viral infection. Nat Rev Immunol 2007;7:753-766

4. Alexopoulou L, Holt AC, Medzhitov R, Flavell RA. Recognition of double-stranded RNA and activation of NF-kappaB by Tolllike receptor 3. Nature 2001;413:732738.

5. Heil F, et al. Species-specific recognition of single-stranded RNA via toll-like receptor 7 and 8. Science 2004;303:1526-1529.

6. Diebold SS, Kaisho T, Hemmi H, Akira S, Reis e Sousa C. Innate antiviral responses by means of TLR7-mediated recognition of single-stranded RNA. Science 2004;303:15291531

7. Hemmi H, et al. Small anti-viral compounds activate immune cells via the TLR7 MyD88dependent signaling pathway. Nat Immunol 2002:3:196-200.

8. Hemmi H, et al. A Toll-like receptor recognizes bacterial DNA. Nature 2000;408:740745 .

9. Yoneyama M, et al. The RNA helicase RIG-I has an essential function in double-stranded RNA-induced innate antiviral responses. Nat Immunol 2004;5:730-737.

10. Yoneyama M, et al. Shared and unique functions of the DExD/H-box helicases RIG-I, MDA5, and LGP2 in antiviral innate immunity. J Immunol 2005;175:2851-2858.
11. Takahasi K, et al. Nonself RNA-sensing mechanism of RIG-I helicase and activation of antiviral immune responses. Mol Cell 2008;29:428-440.

12. Kawai T, et al. IPS-1, an adaptor triggering RIG-I- and Mda5-mediated type I interferon induction. Nat Immunol 2005;6:981-988

13. Xu LG, Wang YY, Han KJ, Li LY, Zhai Z, Shu $\mathrm{HB}$. VISA is an adapter protein required for virus-triggered IFN-beta signaling. Mol Cell 2005; 19:727-740.

14. Seth RB, Sun L, Ea CK, Chen ZJ. Identification and characterization of MAVS, a mitochondrial antiviral signaling protein that activates NF-kappaB and IRF 3. Cell 2005;122:669682. 
15. Meylan E, et al. Cardif is an adaptor protein in the RIG-I antiviral pathway and is targeted by hepatitis $C$ virus. Nature 2005; $437: 1167-$ 1172.

16. Kato H, et al. Differential roles of MDA5 and RIG-I helicases in the recognition of RNA viruses. Nature 2006;441:101-105.

17. Kato H, et al. Cell type-specific involvement of RIG-I in antiviral response. Immunity 2005;23:19-28.

18. Gitlin L, et al. Essential role of mda-5 in type I IFN responses to polyriboinosinic: polyribocytidylic acid and encephalomyocarditis picornavirus. Proc Natl Acad USA 2006;103:8459-8464.

19. McCartney SA, Thackray LB, Gitlin L, Gilfillan S, Virgin HW, Colonna M. MDA-5 recognition of a murine norovirus. PLoS Pathog 2008;4:e1000108.

20. Roth-Cross JK, Bender SJ, Weiss SR. Murine coronavirus mouse hepatitis virus is recognized by MDA5 and induces type I interferon in brain macrophages/microglia. J Virol 2008;82:9829-9838.

21. Foy E, et al. Control of antiviral defenses through hepatitis $\mathrm{C}$ virus disruption of retinoic acid-inducible gene-I signaling. Proc Natl Acad Sci USA 2005; 102:29862991.

22. Loo YM, et al. Distinct RIG-I and MDA5 signaling by RNA viruses in innate immunity. J Virol 2008;82:335-345.

23. Fredericksen BL, Gale M Jr. West Nile virus evades activation of interferon regulatory factor 3 through RIG-I-dependent and -independent pathways without antagonizing host defense signaling. J Virol 2006;80:29132923.

24. Shingai M, et al. Differential type I IFNinducing abilities of wild-type versus vaccine strains of measles virus. J Immunol 2007; 179:6123-6133.

25. Kato $\mathrm{H}$, et al. Length-dependent recognition of double-stranded ribonucleic acids by retinoic acid-inducible gene-I and melanoma differentiation-associated gene 5. J Exp Med 2008;205:1601-1610

26. Satoh T, et al. LGP2 is a positive regulator of RIG-I- and MDA5-mediated antiviral responses. Proc Natl Acad Sci USA 2010;107:1512-1517.

27. Samanta M, Iwakiri D, Kanda T, Imaizumi $T$, Takada K. EB virus-encoded RNAs are recognized by RIG-I and activate signaling to induce type I IFN. EMBO J 2006;25:42074214.

28. Rasmussen SB, et al. Herpes simplex virus infection is sensed by both Toll-like receptors and retinoic acid-inducible gene- like receptors, which synergize to induce type I interferon production. J Gen Virol 2009;90:74-78.
29. Grunberg-Manago M, Oritz PJ, Ochoa S. Enzymatic synthesis of nucleic acidlike polynucleotides. Science 1955;122:907-910.

30. Kim DH, Longo M, Han Y, Lundberg P, Cantin E, Rossi JJ. Interferon induction by siRNAs and ssRNAs synthesized by phage polymerase. Nat Biotechnol 2004;22:321325.

31. Hornung V, et al. 5'-Triphosphate RNA is the ligand for RIG-I. Science 2006;314:994997.

32. Pichlmair A, et al. RIG-I-mediated antiviral responses to single-stranded RNA bearing 5'-phosphates. Science 2006;314:997-1001.

33. Pallansch MA, Kew OM, Palmenberg AC, Golini F, Wimmer E, Rueckert RR. Picornaviral VPg sequences are contained in the replicase precursor. J Virol 1980;35:414-419.

34. Habjan M, et al. Processing of genome $5^{\prime}$ termini as a strategy of negative-strand RNA viruses to avoid RIG-I-dependent interferon induction. PLoS ONE 2008;3:e2032.

35. Ishii KJ, et al. A Toll-like receptor-independent antiviral response induced by doublestranded B-form DNA. Nat Immunol 2006; 7:40-48.

36. Ablasser A, Bauernfeind F, Hartmann G, Latz E, Fitzgerald KA, Hornung V. RIG-I-dependent sensing of poly(dA:dT) through the induction of an RNA polymerase III-transcribed RNA intermediate. Nat Immunol 2009;10:1065-1072.

37. Chiu YH, MacMillan JB, Chen ZJ. RNA polymerase III detects cytosolic DNA and induces type i interferons through the RIG-I pathway. Cell 2009;138:576-591.

38. Nallagatla SR, Hwang J, Toroney R, Zheng X, Cameron CE, Bevilacqua PC. 5'-triphosphatedependent activation of PKR by RNAs with short stem-loops. Science 2007;318:14551458.

39. Hausmann S, Marq JB, Tapparel C, Kolakofsky D, Garcin D. RIG-I and dsRNA-induced IFNbeta activation. PLoS ONE 2008;3:e3965.

40. Rehwinkel J, et al. RIG-I detects viral genomic RNA during negative-strand RNA virus infection. Cell 2010;140:397-408.

41. Pichlmair A, et al. Activation of MDA5 requires higher-order RNA structures generated during virus infection. J Virol 2009;83:10761-10769.

42. Malathi K, Dong B, Gale M Jr, Silverman RH. Small self-RNA generated by RNase L amplifies antiviral innate immunity. Nature 2007;448:816-819.

43. Saito T, Owen DM, Jiang F, Marcotrigiano J, Gale M Jr. Innate immunity induced by composition-dependent RIG-I recognition of hepatitis C virus RNA. Nature 2008; 454:523-527.

44. Cui S, et al. The C-terminal regulatory domain is the RNA 5'-triphosphate sensor of RIG-I. Mol Cell 2008;29:169-179.
45. Takahasi K, et al. Solution structures of cytosolic RNA sensor MDA5 and LGP2 C-terminal domains: identification of the RNA recognition loop in RIG-I-like receptors. J Biol Chem 2009;284:17465-17474

46. Lu C, et al. The structural basis of $5^{\prime}$ triphosphate double-stranded RNA recognition by RIG-I C-terminal domain. Structure 2010;18:1032-1043.

47. Wang Y, et al. Structural and functional insights into 5'-ppp RNA pattern recognition by the innate immune receptor RIG-I. Nat Struct Mol Biol 2010;17:781-787.

48. Lu C, Ranjith-Kumar CT, Hao L, Kao CC, Li P. Crystal structure of RIG-I C-terminal domain bound to blunt-ended double-strand RNA without 5' triphosphate. Nucleic Acids Res 2011;39:1565-1575.

49. Li X, et al. The RIG-I-like receptor LGP2 recognizes the termini of double-stranded RNA. J Biol Chem 2009;284:13881-13891.

50. Fitzgerald KA, et al. IKKepsilon and TBK1 are essential components of the IRF3 signaling pathway. Nat Immunol 2003;4:491-496.

51. Hemmi H, et al. The roles of two IkappaB kinase-related kinases in lipopolysaccharide and double stranded RNA signaling and viral infection. J Exp Med 2004;199:1641-50.

52. Häcker H, et al. Specificity in Toll-like receptor signalling through distinct effector functions of TRAF3 and TRAF6. Nature 2006;439:204-207.

53. Sato M, et al. Distinct and essential roles of transcription factors IRF-3 and IRF-7 in response to viruses for IFN-alpha/beta gene induction. Immunity 2000;13:539-548.

54. Honda K, et al. IRF-7 is the master regulator of type-I interferon-dependent immune responses. Nature 2005;434:772-777.

55. Takahashi K, Kawai T, Kumar H, Sato S, Yonehara S, Akira S. Roles of caspase- 8 and caspase-10 in innate immune responses to double-stranded RNA. J Immunol 2006; 176:4520-4524.

56. An $\mathrm{H}$, et al. Phosphatase SHP-1 promotes TLR- and RIG-I-activated production of type I interferon by inhibiting the kinase IRAK1 . Nat Immunol 2008;9:542-550.

57. Diao F, et al. Negative regulation of MDA5 but not RIG-I-mediated innate antiviral signaling by the dihydroxyacetone kinase. Proc Natl Acad Sci USA 2007; 104:11706-11.

58. Moore CB, et al. NLRX1 is a regulator of mitochondrial antiviral immunity. Nature 2008;451:573-577

59. Huang J, Liu T, Xu LG, Chen D, Zhai Z, Shu HB. SIKE is an IKK\&/TBK1-associated suppressor of TLR3 - and virus-triggered IRF-3 activation pathways. EMBO J 2005;24:40184028.

60. Jounai $\mathrm{N}$, et al. The Atg5-Atg12 conjugate associates with innate antiviral immune 
responses. Proc Natl Acad Sci USA

2007;104:14050-14055.

61. Ishikawa H, Barber GN. STING is an endoplasmic reticulum adaptor that facilitates innate immune signalling. Nature 2008;455:674-678.

62. Zhong B, et al. The adaptor protein MITA links virus-sensing receptors to IRF3 transcription factor activation. Immunity 2008;29:538-550.

63. Gack MU, et al. TRIM 25 RING-finger E3 ubiquitin ligase is essential for RIG-I-mediated antiviral activity. Nature 2007;

446:916-920.

64. Friedman CS, et al. The tumour suppressor CYLD is a negative regulator of RIG-I-mediated antiviral response. EMBO Rep 2008;9:930-936.
65. Oshiumi H, Matsumoto M, Hatakeyama S, Seya T. Riplet/RNF135, a RING finger protein, ubiquitinates RIG-I to promote interferon- $\beta$ induction during the early phase of viral infection. J Biol Chem 2009;284:807-817.

66. Arimoto KI, Takahashi H, Hishiki T, Konishi H, Fujita T, Shimotohno K. Negative regulation of the RIG-I signaling by the ubiquitin ligase RNF 125. Proc Natl Acad Sci USA 2007; 104:7500-7505.

67. Gao D, et al. REUL is a novel E3 ubiquitin ligase and stimulator of retinoic-acid-inducible gene-I. PLoS ONE 2009;4:e5760.

68. Arimoto KI, Konishi H, Shimotohno K. UbcH8 regulates ubiquitin and ISG15 conjugation to RIG-I. Mol Immunol 2008; 45:1078-1084.
69. Tang ED, Wang CY. MAVS self-association mediates antiviral innate immune signaling. J Virol 2009;83:3420-8.

70. Onoguchi K, et al. Virus-infection or 5'pppRNA activates antiviral signal through redistribution of IPS- 1 mediated by MFN1. PLoS Pathog 2010;6:e1001012.

71. Yasukawa K, et al. Mitofusin 2 inhibits mitochondrial antiviral signaling. Sci Signal 2009;2:ra47.

72. Bowie AG, Unterholzner L. Viral evasion and subversion of pattern-recognition receptor signalling. Nat Rev Immunol 2008;8:911922.

73. Komuro A, Bamming D, Horvath CM. Negative regulation of cytoplasmic RNA-mediated antiviral signaling. Cytokine 2008;43:350358. 